\title{
CINE EN VIÑETAS Y CÓMIC EN CELULOIDE: RETROALIMENTACIÓN EN EL UNIVERSO DE FRANK MILLER
}

\author{
Javier GARCÍA-CONDE MAESTRE
}

\section{RESUMEN}

Frank Miller revolucionó el lenguaje narrativo de los cómics de superhéroes con una mezcla de influencias cinematográficas que abarcaban desde el cine negro a las películas asiáticas de artes marciales. En la cumbre de su carrera fue tentado por Hollywood y, aunque las primeras experiencias como guionista no cumplieron sus expectativas, el boom de las adaptaciones de cómics al cine le dio una segunda oportunidad, esta vez como director. La alternancia entre ambos medios desde los años noventa le ha permitido utilizar recursos de uno en el otro, y viceversa, así como reciclar elementos argumentales en ambas direcciones.

Palabras clave: Frank Miller, cómic, cine, género negro, ciencia ficción.

Si hay dos nombres en el panorama del cómic norteamericano convencional que han llevado al cinéfilo a profundizar en la obra original adaptada, estos son Alan Moore y Frank Miller. Mientras el primero renegó primeramente de cualquier adaptación potencial de su obra a la gran pantalla, para pasar después a hacerlo de cualquier secuela impresa y finalmente del cómic en general, Frank Miller ha dado su beneplácito para cualquier adaptación e incluso ha coqueteado delante y detrás de las cámaras en adaptaciones propias o ajenas. Seguiremos su carrera, claro ejemplo de posmodernidad, donde las fronteras entre uno y otro medio se llegan a convertir en auténticas puertas giratorias.

Frank Miller siempre tuvo claro que quería ser dibujante de cómics. Además de este medio de expresión, otras aficiones que cultiva durante su juventud y que acabarán condicionando su carrera son el género negro, tanto novelas como cine, y las películas de artes marciales.

Pronto marcharía a Nueva York, y tras varios trabajos esporádicos en distintas editoriales acaba asentándose en Marvel. Allí se sentirá fascinado por el personaje de Daredevil y acaba encargándose, primero como dibujante y muy pronto como autor completo, de su colección. Como esta se encuentra al borde del cierre se le concede carta blanca para hacer lo que se le antoje: desde el principio aleja la colección del género superheroico para narrar historias de guerras entre bandas, asesinos a sueldo, tráfico de drogas y policías corruptos. 
En uno de sus primeros guiones, Daredevil 169, comienzan a reflejarse sus filias cuando parte de la acción transcurre en un cineclub donde se proyecta El halcón maltés, analizada en profundidad por dos cinéfilos que acaban tomados como rehenes. Igualmente, en el número 185, la acción viene narrada en primera persona por Foggy con la retórica propia del cine negro.

En cuanto al apartado gráfico, convierte la colección en un campo de experimentación. Estamos a finales de los años setenta y el cómic norteamericano de superhéroes seguía, con alguna variación, una estructura narrativa de nueve viñetas por página. En el caso de Miller, si la escena es eminentemente vertical utiliza viñetas verticales, si se requiere un silencio incluso eliminará la viñeta. Los personajes ya no se ven encorsetados por los límites de la viñeta.

La narrativa cinematográfica tampoco queda fuera. Tradicionalmente, por un lado, la posibilidad del lector de controlar el ritmo de lectura hacía posible situar a varios personajes en una misma viñeta $y$, por otro, una pelea tampoco tenía que estar totalmente desarrollada. Miller, sin embargo, cuida las escenas de acción, sobre todo las de lucha, estableciendo una coreografía detallada en cada una de ellas, donde cada movimiento en cada viñeta viene condicionado por la viñeta anterior, y condiciona a su vez la siguiente. En esto no solo influye el cine: su admirado Will Eisner ya dominaba esta técnica décadas antes. Sin embargo, es evidente la influencia del cine oriental, incluso en la utilización de viñetas panorámicas, como una pantalla cinematográfica. En las últimas páginas del cómic Lobezno, con guion a cargo de Chris Claremont, la lucha final con el villano representa un storyboard perfecto.

No solo la proporción de la viñeta se ve influenciada por el cine. Aquí vemos la utilización de un trávelin para dirigir la atención del lector a su antojo a lo largo de lo que en realidad sería una gran viñeta (Figura 1).

La época dorada de Miller se caracteriza por un ir y venir entre Marvel y DC redefiniendo personajes y estilos. En DC realiza Ronin, dejando el género negro y pasando a la cienciaficción y potenciando aún más su fervor por la cultura medieval japonesa. Aunque su mayor mérito en DC sería resucitar otro personaje moribundo: Batman. Esta vez no es un personaje secundario, sino uno de los pilares de la editorial. Alan Moore publicaba casi al mismo tiempo el tebeo de superhéroes para adultos definitivo: Watchmen, pero sus personajes, aun basados en otros existentes, eran originales. La revolución de Miller consistió en aplicar esa evolución a un personaje icónico de la cultura pop, lo que llevaría a que los superhéroes en general abandonasen su habitual público adolescente para conquistar poco a poco a un público más maduro. En El regreso del caballero oscuro se contó su última historia, y en Batman: Año uno su origen, dentro del reinicio del Universo DC en los años ochenta. El regreso del caballero oscuro es, sin duda, una de las obras capitales de su autor y considerada aún hoy por muchos como el mejor cómic de Batman.

En Marvel principalmente se encargaría de Daredevil, primeramente con aquella etapa inicial; y, tras su regreso años más tarde, con la célebre saga Born Again, en la que Matt Murdock es vendido por un personaje muy querido, lo que desencadena su pasión, muerte y resurrección simbólicas, mientras otro célebre personaje reniega de él. En definitiva, una manifestación de la educación católica de Miller. Por otra parte, no podemos olvidar el personaje de Elektra, creado por él mismo y a la que, después de matarla en su primera etapa de Daredevil, rescataría en la serie limitada Elektra: Asesina, publicada por el sello Epic junto a Bill Sienkiewicz, con quien ya había colaborado en una novela gráfica de Daredevil. En este sello Epic se publicaban historias con un enfoque más adulto y una mayor 

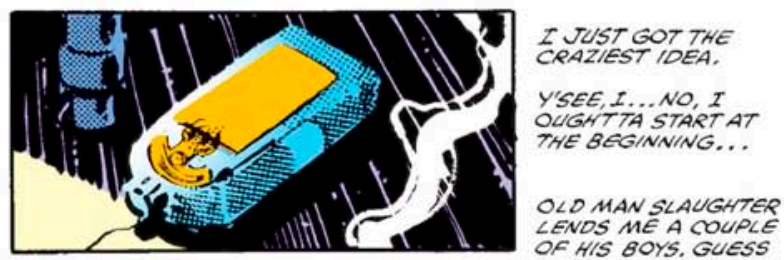

OLO MAN SLAUGHTER OF HIS BOYS. GUESS

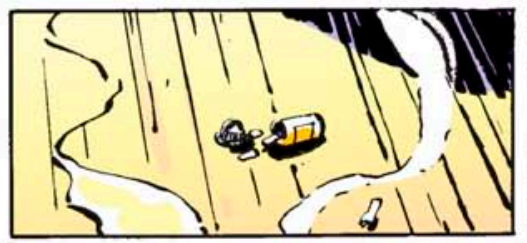

HE'S MORE SCAREO KINGONN FOR

NOW, ANYWAY.

ANYWAY THE BOYS

DGETHER FILES

ON THAT NELSON
SHYSTER AND HIS

PARTNER MATT

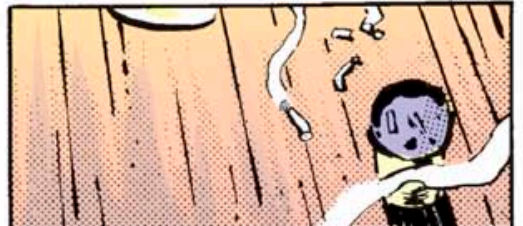

MURDOCK.

SO I SQUIRREL

MYSELF AWAY IN THIS

OO SOME HOMEWORK.

MAYBE IT'S THE BOOZE, OR MAYBE I'M UUST TIRED--
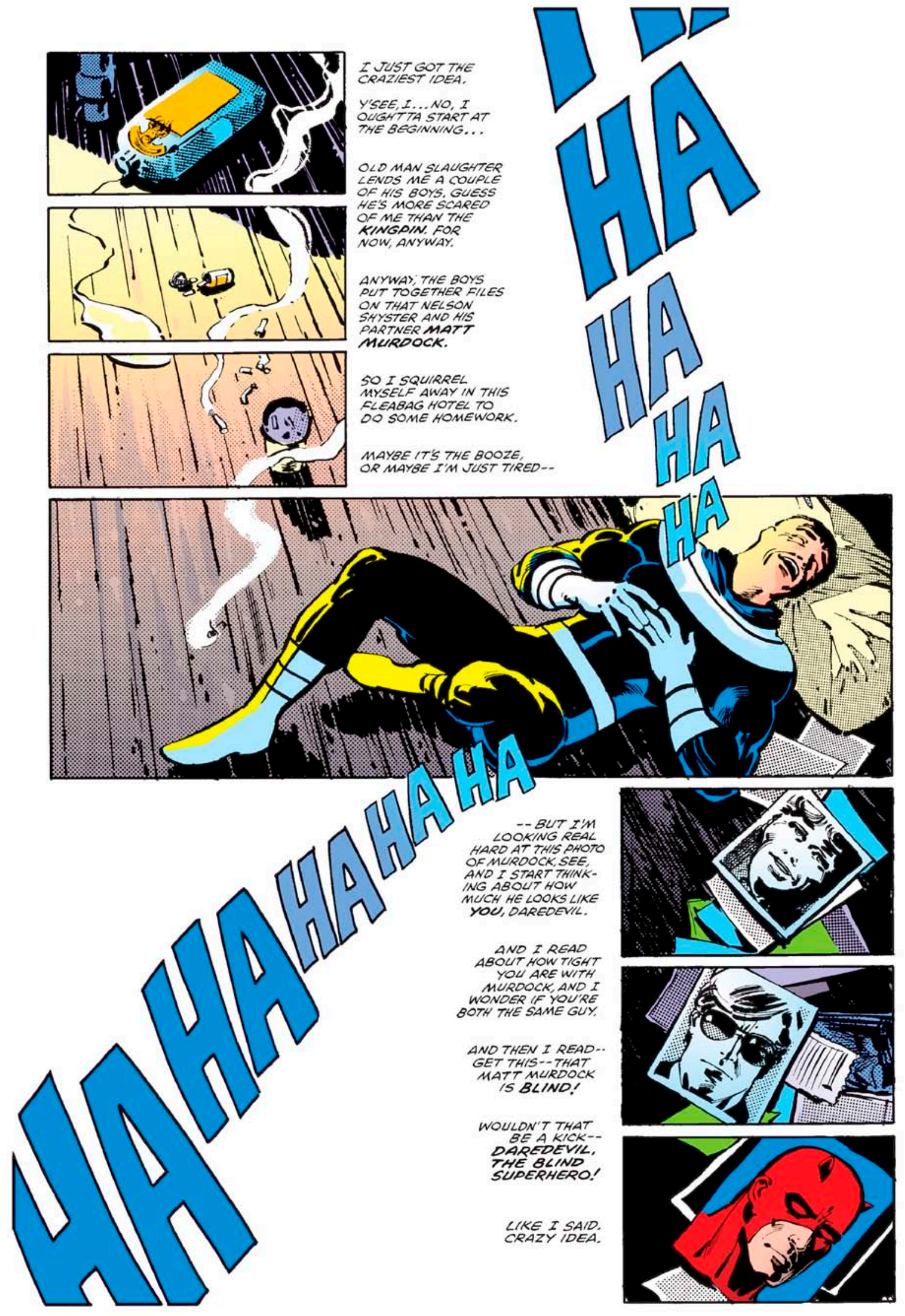

LIKE I SAID.

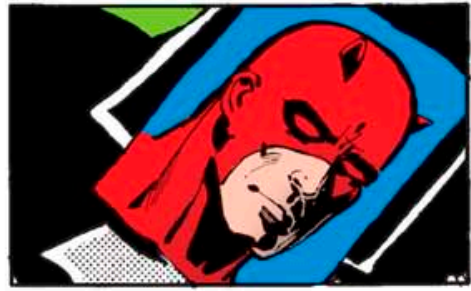

Figura 1. Trávelin cinematográfico traducido a viñetas. (Daredevil USA \#181; 1982) 
calidad técnica, así como algo alejadas de la continuidad Marvel, en caso de narrar historias de superhéroes.

En esta ocasión se narra una historia situada en el pasado de Elektra. Una historia sobre ciborgs psicópatas supuestamente al servicio de las fuerzas del orden desde el punto de vista de uno de estos ciborgs, John Garrett. Garrett fue posteriormente introducido en el universo Marvel convencional, e incluso fue un personaje habitual durante la segunda temporada de la serie de televisión Agentes de S.H.I.E.L.D., interpretado por Bill Paxton, donde se le da una vuelta de tuerca al tema de los implantes cibernéticos.

Al mismo tiempo que se publica Elektra: Asesina, se estrena en los cines una película de bajo presupuesto llamada RoboCop, dirigida por el recién aterrizado desde Holanda Paul Verhoeven y protagonizada por el novato Peter Weller. En ella, un policía muerto en acto de servicio es transformado en un agente de la ley cibernético al servicio de la $\mathrm{OCP}$, empresa privada que gestiona la Policía de Detroit.

La película posee varios puntos en común con trabajos anteriores de Frank Miller. El personaje biónico de Garrett sería lo más obvio, pero coincide aún más con El regreso del caballero oscuro. Por ejemplo, se utiliza en general la televisión de modo similar a como lo hizo Miller, situando al espectador en el contexto sociopolítico en el que transcurre la película. Sorprendentemente, todo esto es casual. En los agradecimientos finales de los créditos no aparece el nombre de Frank Miller y sí el de Howard Chaykin. Chaykin había publicado unos años atrás American Flagg!, donde ya se hacía un uso similar de la televisión, por lo que es más probable que ambos bebieran de la misma fuente.

Aunque sí hay una escena prácticamente idéntica en RoboCop y El regreso del caballero oscuro, y es la situación con rehenes en el ayuntamiento, donde un tal Ron Miller es detenido por RoboCop atravesando una pared para agarrar al villano y que recuerda sospechosamente a la resolución del secuestro del hijo de un millonario en el cómic. (Figura 2).

Casualidades o no, hay además otros puntos que conectan la película con la obra de Miller. Paul Verhoeven afirma que RoboCop es «fascismo para liberales. El filme tiene un punto de vista liberal y lo presenta del modo más violento que se puede imaginar». Sin duda es algo en lo que coincide con varios trabajos de Miller. La lectura a múltiples niveles a partir de una historia en apariencia ligera es otro punto común. Por otra parte, Verhoeven enfoca la historia como una metáfora del nuevo Mesías, contándonos su pasión, muerte y resurrección, como ya hiciera Miller con Daredevil en su Born Again, llegando incluso al final de la cinta a hacerle caminar sobre las aguas.

Sea como fuere, a partir del éxito insospechado de la película, Orion Pictures acelera la producción de la segunda parte. Verhoeven y Numeier recomiendan dejar que se calmen las aguas y hacer algo con calidad. El estudio decide prescindir de ellos y encarga el guion de la secuela a Frank Miller.

Miller acepta y envía un primer acercamiento que es clasificado como «infilmable». Las reescrituras se iban sucediendo, la esencia se fue perdiendo y el guion definitivo seguía sin ser apropiado. Finalmente, el estudio contrató los servicios de Walon Green, escritor de manuscritos tan duros como Grupo salvaje de Sam Peckinpah o Carga maldita de William Friedkin, para escribir un guion final. De esta manera gran parte de la denuncia social tanto de la primera parte como de la idea original de Miller desaparecía en el proceso. Sin embargo, los niveles de violencia inherentes en ambos siguieron presentes, aunque reducidos en parte para mantener la clasificación $\mathrm{R}$ (menores acompañados) en lugar de PG-17 (no autorizada a menores de 17 años). 

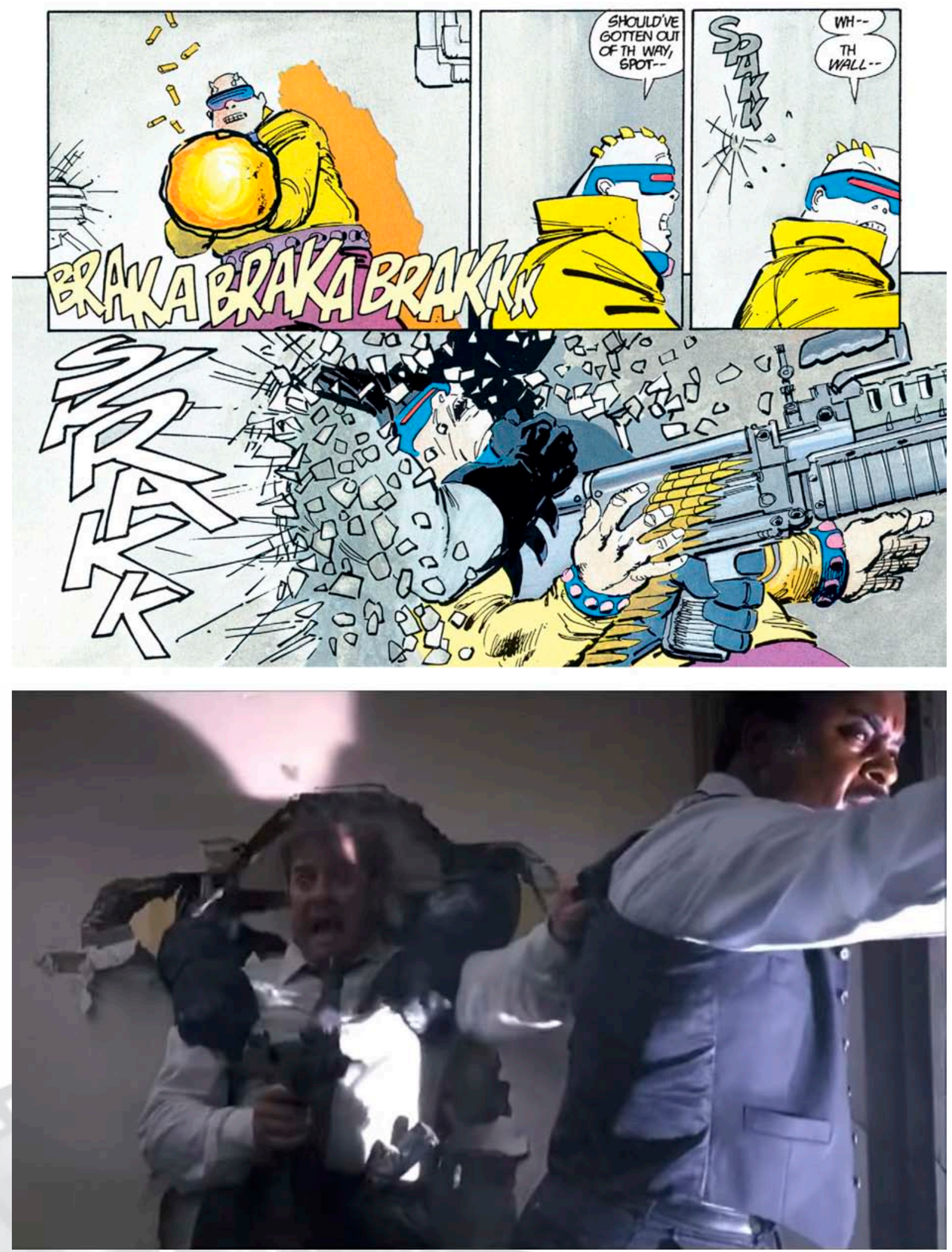

Figura 2. Similitudes casuales entre Return of the Dark Knight (1986) y RoboCop (1987) 
En el resultado final, de todos modos, queda bastante de la marca de la casa de Miller. Asistimos a una abundancia de personajes secundarios tan importantes que RoboCop apenas aparece. Algunos de estos personajes secundarios, de todos modos, son geniales. En particular los villanos.

La OCP decide repetir el éxito de RoboCop, iniciando el proyecto RoboCop 2. Después de varios fracasos con la elección de los policías que compondrían la parte humana, se decide introducir el cerebro de un sádico traficante adicto a la droga de diseño Nuke y utilizar esta droga para poder controlarle mejor, dando lugar probablemente al primer robot drogadicto de la historia. Casi tan interesante es su lugarteniente, un crío con tendencias homicidas realmente terrorífico.

Por otra parte, se aprecian diversas situaciones recicladas de otras obras de Miller. Sobre todo de Elektra: Asesina, como por ejemplo RoboCop desmembrado recordando en varios momentos a Garrett; asistimos a conversaciones sobre comida entre médicos durante las operaciones con pacientes a punto de fallecer; se utilizan las propias partes biónicas para contrarrestar un ataque con tases; se utiliza el cerebro de un psicópata para construir un ciborg con alta capacidad destructiva; el combate final entre el supercíborg y las fuerzas del orden se da durante un acto público, y la destrucción del cerebro se revela como el único modo de acabar con él.

La película fue vapuleada por la crítica y Miller se explayó a gusto sobre lo maltratado que se sentía por el resultado final. Aun así, y sin estar a la altura de su predecesora, es una película inteligente con momentos brillantes, aunque irregular.

Justo después del estreno de RoboCop 2, Miller publica junto a Dave Gibbons Give Me Liberty a través de la editorial Dark Horse, con diversos elementos rescatados directamente de los primeros tratamientos descartados de RoboCop 2. Una historia futurista con notas de denuncia social en la que asistimos a una guerra en el Amazonas y en la que la protagonista, Martha Washington, una niña de raza negra con un don especial para los ordenadores, se ve obligada a alistarse en el P.A.X., un cuerpo militar que le permite borrar su historial delictivo.

En una de las tramas secundarias el presidente, con un sospechoso parecido con el presidente de la OCP de la saga RoboCop, sufre un atentado y se consigue salvar únicamente su cerebro, que será trasplantado a un cuerpo robótico. (Figura 3).

Pero Miller aún estaba lejos de terminar su relación amor/odio con RoboCop. Ya sea para compensarle por el destrozo del guion de la segunda entrega, o porque realmente esperaban reventar la taquilla, Orion Pictures le encarga a Miller el guion de RoboCop 3 antes de llegar a estrenar la segunda entrega con la idea de comenzar a rodar enseguida. Miller vuelve a introducir elementos descartados de su anterior guion y profundiza en la privatización de las fuerzas del orden. La OCP crea el Cuerpo de Rehabilitación, formado por voluntarios cuyo historial delictivo será eliminado si ayudan a desalojar, de manera similar a como se hizo con los judíos, los barrios más desfavorecidos para ser demolidos.

En cuanto a los lugares comunes de Miller, encontramos unos cuantos, como la obsesión por la cultura japonesa presente en casi toda su obra, aquí representada por la fusión OCP/ Kanemitsu; el androide japonés enviado a destruir a RoboCop, que recuerda en muchos aspectos al protagonista de Ronin; el Cuerpo de Rehabilitación, que recuerda al P.A.X. de Give Me Liberty, tanto en uniformes como en política de reclutamiento; la guerra del Amazonas, que ya aparecía en Give Me Liberty; una hacker infantil, como Martha Washington; y un personaje femenino de raza negra llamado Bertha Washington. 

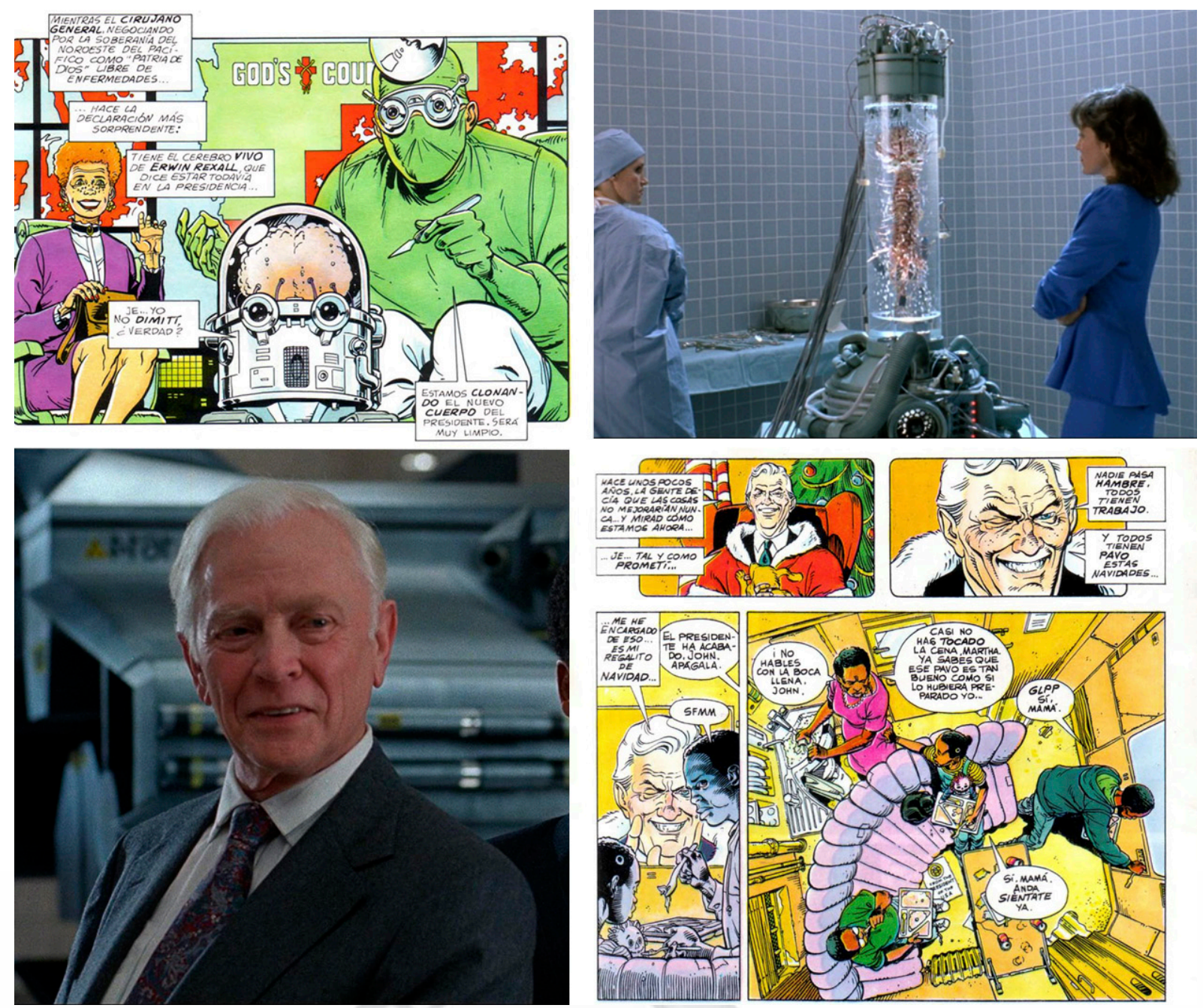

Figura 3. RoboCop 2 (1990) y Give Me Liberty (1990): reciclaje de ideas

Aunque el argumento pueda parecer en principio interesante, asistimos a un auténtico cataclismo. Jon Davison, productor de las dos entregas anteriores, se desvinculó totalmente; así como Peter Weller, su protagonista, que optó por marcharse a rodar El almuerzo desnudo con David Cronenberg. El estudio, al borde de la bancarrota, decidió rebajar su calificación moral de R a PG-13 para aumentar el rango de edad y recaudar más dinero. El humor negro que tenían las dos primeras entregas es sustituido por la autoparodia más burda. Aun así, tardó dos años en poder estrenarse y el batacazo fue tremendo.

El desastre fue tan grande que el defraudado Miller se alejó totalmente de la industria cinematográfica. Incluso años más tarde, en una entrevista a la revista Wizard, como respuesta a la pregunta de si sería posible ver en un futuro adaptaciones de alguna de sus obras, respondió que la única manera que veía era dirigiéndolas él mismo, por lo que no era probable.

Exactamente diez años más tarde del estreno de RoboCop 3, en 2003, la editorial independiente Avatar Press publicó la serie limitada RoboCop, anunciada como la adaptación del guion original de Miller para RoboCop 2. Steven Grant, famoso por escribir la serie limitada El Castigador: Círculo de sangre, ilustrada por Mike Zeck, figura como adaptador secuencial en lugar de como escritor; mientras que el dibujo recae en el español Juan José Ryp. 
Si aceptamos que este era el guion original vemos que, efectivamente, no era filmable. Al menos a finales de los ochenta, y menos para un estudio pequeño. Demasiados escenarios destruidos y efectos especiales. Por no hablar de las innumerables tramas secundarias. En él seguimos dando vueltas al derribo del Viejo Detroit. Al aplicarse los desahucios vemos a RoboCop entrando en conflicto con las directrices «Cumplir la ley» y «Proteger al inocente». Se decide crear un nuevo RoboCop más controlable y con menos integridad ética. Para ello se usa a un agente del Cuerpo de Rehabilitación herido, Kong, un individuo enorme adicto a las anfetaminas con bastantes reminiscencias del personaje de Nuke de Born Again (Nuke era, además, el nombre de la nueva droga ficticia que aparecía en la película final).

Es interesante que en las noticias y en los anuncios aparezca la Corporación Sawa de Ronin; y tanto los productos gigantes Behemot como la refinería en Texas incendiada mediante el proyecto Guerra de las Galaxias finalmente verían la luz en Give Me Liberty. La clave de denuncia de las corporaciones, la indefensión del ciudadano ante los poderes fácticos y la decadencia de la sociedad a través de la televisión son efectivamente mayores que en la versión final.

Posteriormente, la editorial Boom! haría lo mismo con el guion de RoboCop 3 en la colección RoboCop: Último asalto, de nuevo con Grant a los textos y el turco Korkut Öztekin a los lápices. La historia se sitúa tiempo después de la segunda entrega, con su compañera Lewis ya fallecida y Murphy viviendo al margen de la ley, impidiendo los desahucios, autorreparándose en un almacén abandonado. Allí es localizado por fin por Marie, una experta en tecnología e informática obsesionada con el logro de RoboCop que lleva tiempo buscándole. La trama conducirá a la destrucción de RoboCop y la creación de un nuevo cuerpo, más avanzado. Por su parte, Marie será asesinada, pero sus pautas cerebrales toman el control de los ordenadores de la OCP y termina en un cuerpo robótico.

La historia en sí sigue estando muy alejada de las mejores obras de Miller, aunque comparada con la película es una mejora considerable. El personaje de Marie, que acabaría siendo desdoblado en la repelente niña informática y la doctora Lazarus, no tiene un nombre elegido al azar, e incluso luce una cruz al cuello en todas y cada una de sus representaciones (Figura 4). Se produce un proceso de calvario, muerte y resurrección similar, aunque más físico, al de Born Again, continuando la alegoría religiosa comenzada en la primera entrega por Verhoeven.

Pero aún quedaría más material sobre el policía cibernético. Mientras RoboCop 3 permanecía en el limbo esperando a poder ser estrenada, y demostrando que aún albergaba esperanzas para el personaje, Miller publica Robocop versus Terminator junto a Walter Simonson. Aprovechando que los derechos de ambas películas pertenecían a Orion Pictures se lanzó este pastiche, algo muy habitual en la época (Aliens vs. Predator fue el más famoso), y que fue además la base de un videojuego bastante popular en su momento.

Con una premisa cogida por los pelos asistimos a una serie de sucesivos viajes en el tiempo hacia adelante y hacia atrás. Con cada viaje se va liando la madeja, unas veces la victoria es para unos, otras veces para otros. Por el camino se van alterando ambas líneas temporales saltándose a la torera, de paso, cualquier regla sobre las paradojas temporales. Miller demuestra con su trabajo en RoboCop que domina las claves del género negro, pero la ciencia-ficción dura no es, definitivamente, su campo.

Tras el desastre en su intento de introducirse en Hollywood, Frank Miller vuelve como autor completo al mundo del cómic. Y no podría haber una opción más lógica que los 

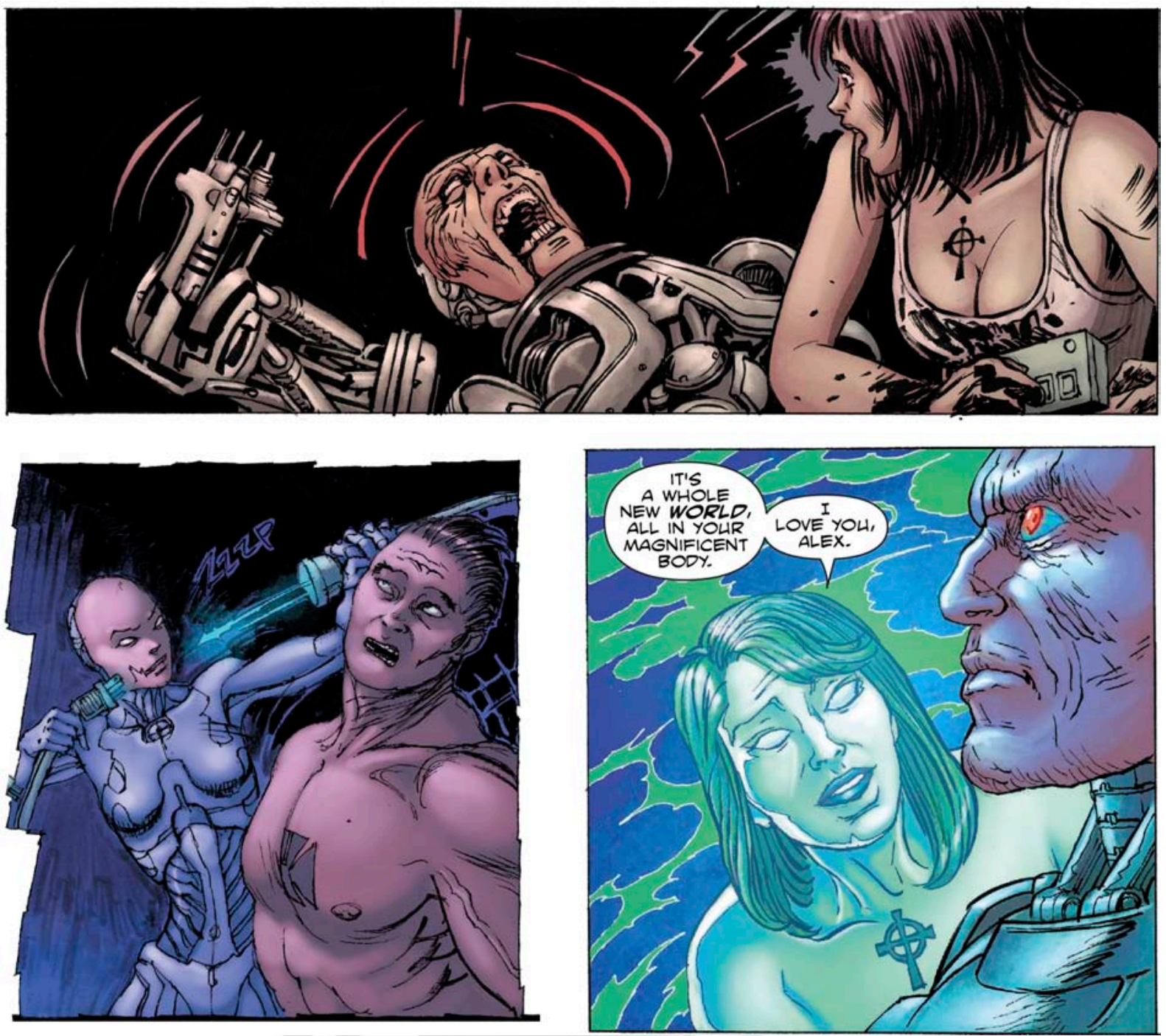

Figura 4. Las diversas encarnaciones de Marie en RoboCop: Último asalto (2013)

personajes de Matt Murdock y Elektra, con Elektra Lives Again y El hombre sin miedo, ésta junto a John Romita Jr.

Siempre se ha dicho de esta última colección que era un guion no utilizado para una película, aunque no sea exactamente así. Sí es cierto que a Miller se le pidió, a finales de los ochenta, lo que se llama un plot (una base) para una serie de televisión sobre el personaje que no llegó a realizarse. Al mismo tiempo, Romita le expresó su interés en trabajar juntos y, puesto que seguía dándole vueltas a esa historia no utilizada, decidieron hacer una «biblia» de Daredevil: actualizar el origen, un tanto caótico con las sucesivas revisitaciones, desde el que partir de cero en la colección. Pero el salto a Hollywood se interpuso en el camino y la serie se había postergado hasta entonces.

Y así llegamos a las dos obras más importantes de su retorno al mundo del cómic: Sin City y 300. En 1998, publica este homenaje a la película de 1962 El león de Esparta, de Rudolph Maté, sobre la batalla de las Termópilas. Incluso el extraño formato horizontal de la obra es un homenaje al formato panorámico del CinemaScope. Miller afirma que fue esta historia la que le descubrió la verdadera naturaleza del héroe: alguien que no siempre gana y que en ocasiones tiene que arriesgarlo todo por sus ideales. 
Son numerosas las referencias a este momento histórico en su obra anterior: en El regreso del caballero oscuro aparece una actriz pornográfica que se llama Hot Gates (la denominación anglosajona para las Termópilas), se nombraba en el guion de RoboCop 2, lo cita Martha Washington, es clave en el desenlace de Sin City: La gran masacre, y en una alucinación en Sin City: Ida y vuelta al infierno uno de los personajes es percibido como Leónidas con una metralleta. Era lógico que antes o después acabara adaptándolo.

Pero, sin duda, su obra cumbre en esta época será Sin City, que comenzó a publicarse en 1991. Nos dejamos de homenajes e influencias para entrar de lleno y abiertamente en el género negro, fusionando tanto su tradición escrita como cinematográfica. Una primera obra por entregas, en la mejor tradición de las novelas pulp, es seguida por diversas miniseries cuyos personajes se entrecruzan. Los rumores de adaptaciones al cine se suceden con intermitencia prácticamente desde la publicación de la primera historia.

Sin City se alargaría hasta 1999, justo en un momento en el que sucede algo que cambiaría la relación cómic/cine: Marvel se encuentra al borde de la bancarrota y para poder salvar la empresa reparte los derechos de autor de casi todos sus personajes entre los grandes estudios de Hollywood.

Daredevil queda en manos de 20th Century Fox, junto a X-Men y Los 4 Fantásticos, contando las tres con un presupuesto bastante reducido. El director elegido para Daredevil fue Mark Steven Johnson. Cuando este presentó su película al estudio, apoyado por Avi Arad (entonces responsable de la división cinematográfica de Marvel) el estudio decidió cortarlo, dada su excesiva duración: 120 minutos. El productor Gary Foster afirmó que «prefería velocidad antes que escenas bien filmadas, interpretadas o dirigidas que iban a bajar el ritmo de la película. [...] El contenido intelectual no es tan importante como la experiencia emocionante y rápida de este tipo de películas».

Por lo tanto, se le quitaron a la película unos treinta minutos y se centraron en la acción. Se mutiló lo concerniente al personaje de Kingpin (el villano), al de Elektra (la chica), al de Ben Urich (una trama que amenaza con sacar a la luz la identidad secreta del héroe) y se eliminó por completo una trama secundaria protagonizada por Foggy (amigo, socio y compañero de investigación), que unificaba el resto de tramas. Además, dado que las escenas de lucha no hubiesen permitido una calificación PG-13, se suavizaron y recortaron. Así que, en definitiva, tenemos una película sin argumento ni desarrollo de personajes para poder ver una cinta de acción sin peleas.

Mucho más interesante resulta la versión del director aparecida en formato doméstico más tarde. Tampoco es que ponga la película a la altura de Casablanca, no nos engañemos, pero es cierto que es de esas raras ocasiones en las que el montaje alternativo es ciertamente otra película totalmente diferente, con una considerable mejora, y no un simple sacacuartos.

Johnson no se anda con rodeos y adapta la etapa de Miller al frente de la colección. Nos cuenta el origen del personaje, nos muestra su trabajo como abogado de la Cocina del Infierno, su relación con Foggy (Jon Favreau es de lo mejor de la película), cómo aplica su propia justicia cuando no puede hacerlo en los tribunales, cómo ambas facetas se unen en el intento de sacar a la luz al misterioso Kingpin, introduce al personaje de Elektra y se inicia la imposible historia de amor, entra en escena Bullseye y llegamos al final que todos conocemos.

Lorenzo Díaz afirmó en el prólogo de la primera edición española de El hombre sin miedo de Miller y Romita de Forum: «este trabajo, en lugar de contar una sola historia cuenta dos y ese mérito ha acabado por perjudicarle» (DÍAZ: 1994, 3). Ese mismo error se 
aplica a la película, ampliado, puesto que mientras aquel contaba solo el principio de ambas historias en 150 páginas aquí quiere contar el principio y el final, y la ajustada duración de una película no da más de sí.

La película tiene otros defectos. En cuanto al reparto, Ben Affleck fue el más vapuleado por los fans, aunque el actor que realmente falla es Michael Clarke Duncan, no porque fuese un actor negro como se criticó en su momento por los más puristas, lo que francamente resulta indiferente; sino porque su interpretación retorna al malo de tebeo de otras épocas y no resulta amenazador, a pesar de su tamaño.

Los efectos digitales tampoco son de lo mejor del cine de superhéroes, pero hay que ponerse en situación: recordemos el presupuesto mínimo de la película y la época en la que nos encontramos. Si bien es cierto que cuando se utilizan actores reales la cosa mejora muchísimo. La pelea inicial (sin censurar) en el bar de Josie podría enseñar al afamado Christopher Nolan un par de cosas en cuanto a cómo dirigir escenas de acción.

A pesar de todos sus defectos, la película no es tan mala como la fama que le precede, y es entretenida y respetuosa con el personaje. El propio Miller estaba bastante contento con muchos aspectos del montaje original; y es recomendable que aquel que no haya visto el montaje del director le eche un vistazo antes de volver a opinar de nuevo sobre ella.

Después del fracaso de la película en las salas se descartó una secuela directa y se optó por darle una película en solitario a Elektra. Años más tarde, Jennifer Garner diría que la hizo porque su contrato la obligaba, pero siendo consciente de lo horrible que era.

Un par de años después de Daredevil Robert Rodríguez, tal vez recordando aquella entrevista de Wizard, consigue convencer a Miller para dirigir a cuatro manos la adaptación de Sin City. Se haría todo en pantalla verde, tratando el color para convertirlo en blanco y negro y añadiendo los fondos digitalmente. El maquillaje y la utilización de fondos convierten la película en una experiencia interesante tanto para aficionados a la obra original como para extraños.

Esto, realmente, no es diferente de lo que se hizo con Mary Poppins cuarenta años antes. El mismo principio con nueva tecnología. Ni siquiera en su momento era pionera: Sky Captain y el mundo del mañana ya se había estrenado un año antes, rodada de la misma manera pero recreando los seriales de los años cuarenta en lugar de los cómics.

En los créditos no aparece ningún escritor o guionista, solo se especifica: «Basado en la novela gráfica de Frank Miller». La idea era utilizar los cómics como storyboard y recrear plano a plano el cómic (Figura 5). Precisamente la mayor virtud de la película se convierte en su mayor defecto para algunos de sus seguidores: no aporta nada nuevo.

Dado que una sola historia no hubiera cubierto la duración de una película convencional, se decide entrelazar varias de ellas. No deja de ser curiosa la elección de series. Por supuesto, El duro adiós, la historia inicial que desencadenó todo, es una de ellas; junto a Ese cobarde bastardo, consideradas ambas las dos mejores historias. Pero para cerrar el trío se escoge La gran masacre, secuela de menos calidad de Moriría por ella, que se deja premeditadamente para la segunda entrega. La acertada elección del reparto se sumaba a los aciertos y daba como resultado final una de las mejores adaptaciones del cómic al cine.

Nueve años más tarde se lanzó una secuela tardía que adaptaba, como siempre se dijo, Moriría por ella, e incluía dos historias totalmente nuevas. El mayor inconveniente era que las historias que quedaban por adaptar no estaban a la altura de las iniciales, pero aun así son mejores que lo que escribió el mismo Miller para completar la película. 




Figura 5. Sin City (2005): cómo utilizar un cómic como storyboard de su propia adaptación

El considerable retraso añadía el inconveniente de que varios de los actores habían fallecido y otros tuvieron que ser sustituidos por problemas de agenda. Si tenemos en cuenta este problema, añadimos el tema del guion y el hecho de que el maquillaje esta vez fuera horrible, tenemos como resultado final un producto que no está a la altura de la primera entrega, lo que hizo (tal vez injustamente) que solo haya llegado a nuestro país mediante plataformas de video bajo demanda, y en fechas recientes.

El boom de adaptaciones de superhéroes ya se había desatado y DC no quiere quedarse atrás, por lo que activa un proyecto para relanzar a su personaje estrella: Batman. La franquicia había caído en el olvido después del desastre de Batman y Robin. Lo cierto es que la serie no había bebido demasiado de la obra de Miller, pero el nuevo proyecto, a cargo de Darren Aronofsky, pretendía partir del potente Batman: Año uno, con guion del mismísimo Miller, de nuevo bien avenido con el séptimo arte. Este proyecto pretendía reinventar el personaje y mostrar algo nuevo. Tanto, que se eliminaban prácticamente todos los puntos que definían tanto a Batman como a Bruce Wayne.

Finalmente, los tira y afloja entre Aronofsky y Miller hicieron embarrancar el proyecto, que fue retomado por Christopher Nolan y renombrado como Batman Begins. La película parte del trabajo de Miller, así como de muchos otros acercamientos a los primeros años del personaje, e incluso escenas de la película de animación Batman: La máscara del fantasma, de Bruce Timm. 
Dos años después de Batman Begins se toma otra de sus obras icónicas de los últimos años para llevarla a la gran pantalla: 300, en la que el propio Miller colaboraba como productor. La cinta sigue fielmente el argumento del cómic pero, ciertamente, la serie de Miller es de lectura muy rápida, por lo que se añade una subtrama con la reina Gorgo intentando conseguir el apoyo del senado espartano para su marido, dando peso al personaje femenino y potenciando la relación con su marido.

Si esa variación con respecto a la obra original es más que justificable, no lo son en absoluto otros añadidos que hace el director y que desvirtúan el concepto. En ningún momento se dice que el cómic sea un ensayo histórico y se toma bastantes licencias en algunos detalles, pero es cierto que Miller llevó a cabo un gran trabajo de documentación y ambientación desvirtuados con múltiples anacronismos en pos del efectismo que, junto a los toques de cine de terror de Zack Snyder, que venía de rodar Amanecer de los muertos, sobran.

En su momento se acusó de haber realizado una película xenófoba y racista que atacaba al pueblo iraní. Aunque con los años, y sobre todo a partir del 11-S, Miller se ha radicalizado en sus posturas, tanto en el cómic como en la película 300 vemos la representación de un hecho histórico con dos bandos enfrentados, narrado desde el punto de vista de uno de ellos, como siempre se ha hecho y se hará en literatura y cine, y exagerado como cualquier narración subjetiva. En ella, un pueblo intenta defenderse de una invasión real; intentar encontrar paralelismos entre la Persia de entonces, siglos antes del Islam, y el Irán de hoy resulta demasiado paranoico. Aunque es cierto que en estos tiempos de corrección política desmesurada, algo de paranoia siempre flota en el ambiente.

Dado el éxito de la colección, Miller se había comprometido a publicar una secuela. Llegó a terminar tres números, pero no acabó publicándose hasta 2018. Ante el inesperado éxito en taquilla la productora no quería esperar y se lanzó a rodar una secuela anunciada como «Basada en la novela gráfica de Frank Miller». En realidad cogía el mismo hecho histórico que debería haberse narrado en la serie y se escribió desde cero, dando lugar a 300: El origen de un imperio.

En 2008, y dado el éxito de Sin City y 300, Miller debuta como director en solitario aunque en lugar de optar por adaptar una de sus propias obras decide homenajear a su maestro Will Eisner rodando una adaptación de su mítica creación The Spirit. Utilizando la misma técnica de pantalla verde, lo que hace Miller realmente es narrar una historia más propia de Sin City, en lugar de respetar la iconografía propia del personaje. En su intento de crear una atmósfera atemporal introduce anacronismos como móviles, portátiles, helicópteros y armas modernas, creando un resultado final totalmente inverosímil.

Hay muchos planos homenaje, referencias a portadas de la colección, perspectivas. Guiños a Kurosawa, Leone, Kubrick... Pero todo queda en la estética, no en la esencia o la narrativa. Gran narrador como ha sido en el mundo del cómic, comete el mayor error de un adaptador al no comprender que lo que funciona en un medio no siempre funciona en el otro.

Pero aún quedaban clásicos por adaptar. Un año antes, Warner había fundado el sello DC Universe desde el que producían largometrajes de animación. Coincidiendo con el capítulo final de la saga de Nolan se lanzó Batman: Año uno. La película sigue casi palabra por palabra lo contado por Miller en su célebre historia. El mayor problema de la película, sin embargo, es el hecho de volver a contar, otra vez, un origen que nos sabemos de memoria y del que ya habíamos visto una buena parte en la versión que se estaba viendo en esos momentos en la gran pantalla. 
Lanzar esta película, aunque sonara a reiterativo, sin embargo, no suponía una casualidad. La idea era abrir boca para el proyecto más ambicioso del estudio hasta la fecha: la adaptación completa de El regreso del caballero oscuro en un díptico lanzado con apenas cuatro meses de diferencia entre cada entrega. No era, de todos modos, la primera vez que Bruce Timm adaptaba esta obra, aunque solo fuera algún fragmento. En un capítulo de la serie Las nuevas aventuras de Batman y Robin se nos daba una visión muy oscura, en la que se narra la derrota del líder mutante a manos de Batman, precedida por aquel momento ya comentado en el que Batman atraviesa una pared para atrapar a los secuestradores. La voz de Batman la pone Michael Ironside, que originalmente iba a ser RoboCop, pero era demasiado alto y grande para añadirle la armadura.

Y cuando llega la hora de elegir al reparto para encarnar a este Batman crepuscular se cierra el círculo que comenzó en 1987. Qué mejor actor para protagonizar esta obra que Peter Weller, RoboCop, terminando así de entrelazar a estos dos personajes con tantos puntos en común. $Y$ vuelve a interpretar la escena que ya apareciera en aquella película, atravesando la pared para evitar un secuestro, planteando la típica pregunta: «¿Qué fue antes, el huevo o la gallina?».

La producción de la nueva adaptación completa sigue la trama más o menos fielmente, pero elimina la voz en off, los textos de apoyo que tantos matices aportan a la obra de Miller. Por otra parte, con la mejora de las técnicas de animación este estudio de animación ha ido dotando de un gran dinamismo a las escenas de acción, a años luz de la rigidez y
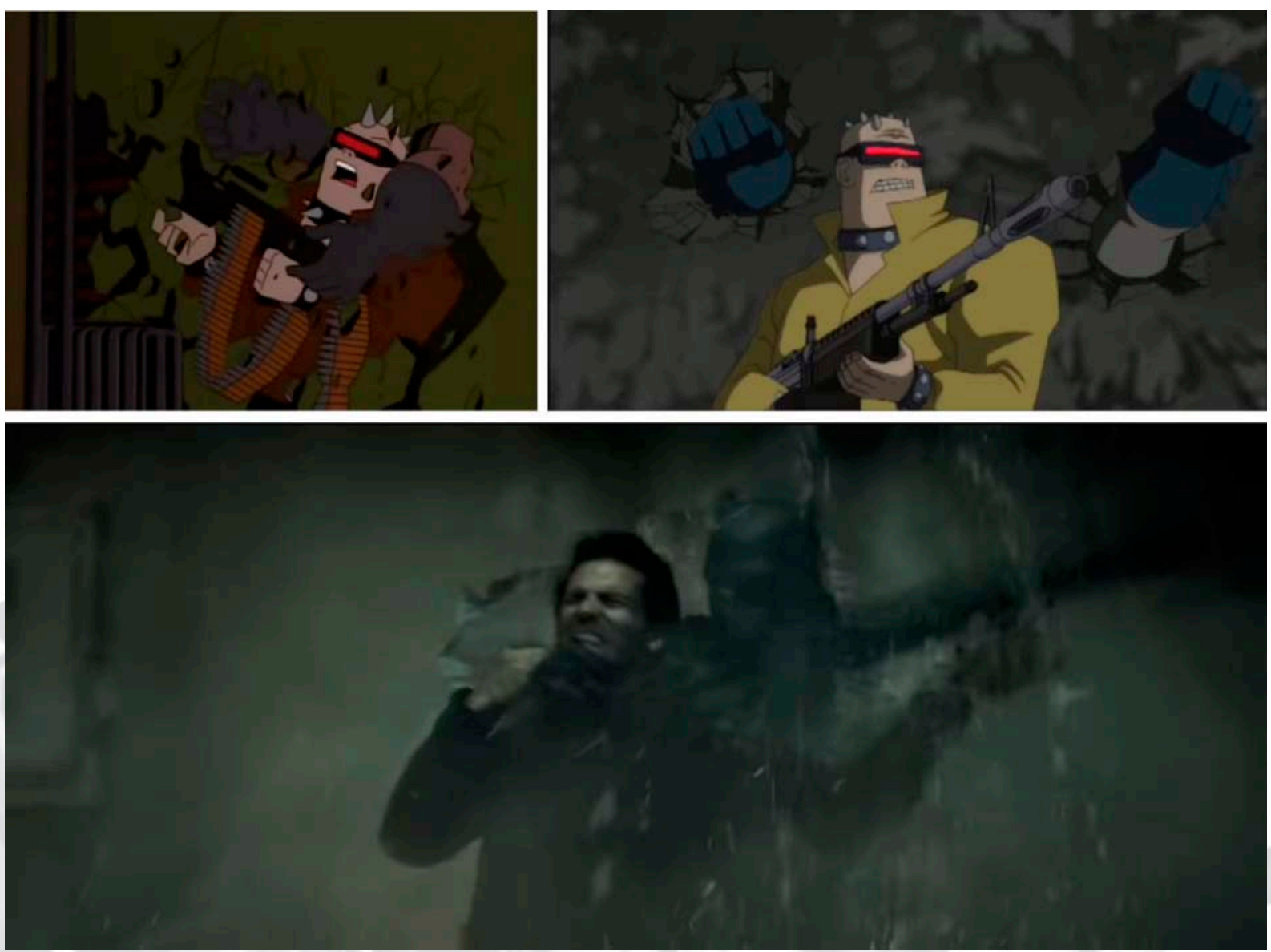

Figura 6. (De izquierda a derecha y de arriba a abajo): Las nuevas aventuras de Batman (1997), El regreso del caballero oscuro (2012) y Batman v Superman: El amanecer de la justicia (2016) 
el acartonamiento de los años ochenta y noventa. Esta mejora se ha convertido en defecto con el paso de los años, con peleas cada vez más alargadas que, en El regreso del caballero oscuro, llegan a ser en su mayoría tediosas. Aun así, dada la amplitud de la obra original, es una adaptación más que digna y que seguramente será lo más fiel que vayamos a ver nunca.

En el año 2016 se estrenó Batman v Superman: El amanecer de la justicia, dirigida por Zack Snyder (el responsable de 300) y escrita por David Goyer, responsable de los guiones de la trilogía de Batman de Nolan. El argumento principal coge básicamente las últimas páginas de El regreso del caballero oscuro, pero sacadas de contexto; de modo que visualmente puede recordarnos al original, pero no en esencia. A su alrededor construye el resto de la historia, insertando otros momentos de la citada obra como, en un alarde de originalidad y capacidad de sorpresa, la escena en la que Batman atraviesa la pared y que ya hemos nombrado aquí multitud de ocasiones (Figura 6).

Y así llegamos al momento actual, con otra película de Batman en rodaje, por fin, tras numerosos problemas. Mientras el proyecto incluía a Ben Affleck como director-escritoractor parecía que Miller volvería a ser la influencia más directa. Pero con el cambio como director-escritor por Matt Reeves parece que se ha girado más hacia una historia neo noir de detectives y no de superhéroes (por fin, por otra parte) que va a beber de las mismas fuentes de las que bebía Miller sin seguirle a él directamente.

La buena estrella de Miller en el mundo del cómic empezó a declinar después de su primer contacto con el cine y, salvo Sin City y 300, ninguna de sus obras tuvo la repercusión de sus trabajos de los años ochenta. Sin embargo, una nueva faceta surgió ante él con el boom de las adaptaciones de cómics, tal vez arruinada también por el desastre que supuso The Spirit. Esperemos que en algún momento, sea en el medio que sea, este hombre que tantos buenos momentos nos ha hecho pasar y tantas imágenes icónicas ha creado recupere el pulso narrativo. Lo que no se puede negar es la capacidad de fascinación que aún genera ni que jugó, a ambos lados de la ecuación, un papel clave en la relación cómic y cine y en el boom de adaptaciones que vivimos actualmente.

\section{BIBLIOGRAFÍA}

[s.f.] (1996/07). Wizard: The Guide to Comics n 59, Wizard Press.

CHAYKIN, H. (1983-1989): American Flagg! n 1-14, Illinois, First Comics.

Claremont, C., Miller, F., Rubinstein, J., Wein, G. (1982): Wolverine Vol. 1 n 1-4, Nueva York, Marvel Comics.

DÍAZ, L. (1994), «Prólogo» El hombre sin miedo, Colección One-Shot n 5, Barcelona, Forum, p. 3.

Grant, S., Duffy, J., Zeck, M., Vosburg, M., Beatty, J., Sharen, B. (1986): «El Castigador: Círculo de sangre» El Castigador Vol. 1 n 1-5, Nueva York, Marvel Comics.

Grant, S., ÖZTEKIN, K., SHALVEY, D. (2013-2014): RoboCop: último asalto n 1-8, Los Angeles, Boom! Studios.

Grant, S., Ryp, J. J., McKinley, A., Waller, G., SWeeney, M. (2003-2006): RoboCop n 1-9, Illinois, Avatar Press.

MiLler, F. (1992): Sin City: El duro adiós, Milwaukie, Dark Horse Comics.

MILLER, F. (1993-1994): Sin City: Moriría por ella n 1-6, Milwaukie, Dark Horse Comics.

MilleR, F. (1994-1995): Sin City: La gran masacre n 1-5, Milwaukie, Dark Horse Comics. 
Miller, F. (1996): Sin City: Ese cobarde bastardo, Milwaukie, Dark Horse Comics.

MilleR, F., GibBONS, D., SMITH, R. (1990-1991): Give Me Liberty n² 1-4, Milwaukie, Dark Horse Comics.

Miller, F., JANSON, K., VARLEY, L. (1986): El regreso del Caballero Oscuro no 1-4, Nueva York, DC Comics.

Miller, F., JANSON, K., Wein, G. (1981): Daredevil Vol. 1 n 169, Nueva York, Marvel Comics. Millerer, F., JANSON, K., WeIN, G. (1982): Daredevil Vol. 1 n 181, Nueva York, Marvel Comics. Miller, F., JANSON, K., WeIN, G. (1982): Daredevil Vol. 1 n 185, Nueva York, Marvel Comics. MilleR, F., MAZZUCCHELLI, D., LeWIS, R. (1987): «Batman: Año uno» Batman Vol. $1^{\circ}$ 404-407, Nueva York, DC Comics.

Miller, F., Mazzucchelli, D, Scheele, C., LeWIS, R. (1986): «Born Again», Daredevil Vol. $1 n^{\circ}$ 227-233, Nueva York, Marvel Comics.

Miller, F., Romita JR., J., Williamson, A., Scheele, C. (1993-1994): El hombre sin miedo $n^{\circ}$ 1-5, Nueva York, Marvel Comics.

MilLeR, F., SiENKIEWICZ (1986-1987): Elektra: Asesina no 1-8, Nueva York, Marvel Comics.

MILLER, F., SimOnSON, W. (1992): RoboCop vs Terminator, Milwaukie, Dark Horse Comics.

MILLER, F., VARLEY, L. (1983-1984): Ronin nº 1-5, Nueva York, DC Comics.

Miller, F., VARLEY, L. (1990): Elektra Lives Again, Nueva York, Marvel Comics.

Miller, F., VARLEY, L. (1998): 300 n 1-5, Milwaukie, Dark Horse Comics.

Miller, F., VARLEY, L. (1999-2000): Sin City: Ida y vuelta al infierno n 1-9, Milwaukie, Dark Horse Comics.

Miller, F., Varley, L. (2018): Xerxes n¹-5, Milwaukie, Dark Horse Comics.

Stradley, R., Norwood, P., Wagner, C., Story, K., Campanella, R. (1990-1991): Aliens vs. Predator Vol. 1 n 1-4, Milwaukie, Dark Horse Comics.

\section{Filmografía}

\section{A) Cine}

(2004) Giving the Devil His Due: The Making of the 'Daredevil' Director's Cut, EE.UU., 20th Century Fox.

Abraham, M., Bernstein, A., Bliss, T. A., Jones, D. E., Messina, M. D., Newman, E., Rubinstein, R. P. (productores), SNYDER, Z. (director). (2004). Amanecer de los muertos (Dawn of the Dead). EE.UU., Japón, Canadá, Francia, Strike Entertainment, New Amsterdam Entertainment, Metropolitan Filmexport, Toho-Towa.

Addie, S., Arad, A., Feige, K., Foster, G., Johnson, M. S., Lee, S., Mclaglen, J., Milchan, A., O'CONNOR, B. (productores), BOWMAN, R. (director). (2005). Elektra (Elektra), EE.UU., 20th Century Fox, Regency Enterprises, Marvel Enterprises, New Regency Pictures, Horseshoe Bay Productions, Epsilon Motion Pictures, Elektra Productions, SAI Productions.

Andrick, J., Burns, M., Del Prete, D., DeSAnto, F. J., Lischak, B., Maier, S., McDonough, L., Melniker, B., PAseornek, M., Pritzker, G., Sadeghi, M., Uslan, M. E., Walpole, A. (productores), MILLER, F. (director). (2008). The Spirit (The Spirit), EE.UU., Lionsgate, Dark Lot Entertainment, MWM Studios, Continental Entertainment Group (CEG).

Arad, A., Atlan, N., Barnathan, M., Cleary, L., Elchinger, B., Fanger, R., Feige, K., Gorder, D., LeE, S., RAdClifFe, M., Williams, K., WINTER, R. (productores), StORY, T. (director). (2005). Los 4 fantásticos (Fantastic Four), EE.UU., Alemania, Twentieth Century Fox, 
Constantin Film, Marvel Enterprises, 1492 Pictures, Bernd Eichinger Productions, Kumar Mobiliengesellschaft mbH \& Co. Projekt Nr. 3 KG.

Arad, A., Courtney, Cross Trujillo, B., Beige, K, Foster, G., Lee, S., Milchan, A., Williams, Bernard, Winther, K. H., Carraro, B., Devan, B. (productores), Johnson, M. S. (director). (2003). Daredevil (Daredevil). 20th Century Fox, Marvel Enterprises, New Regency Pictures, Horseshoe Bay Productions, Epsilon Motion Pictures, Regency Enterprises.

Arad, A., Desanto, T., Donner, R., Edelman, M., Feige, K., Lee, S., Nimerfro, S., Shuler DONNER, L., SIMON, J., TODMAN JR., B., WINTER, R. (productores), SINGER, B. (director). (2000). X-Men (X-Men), EE.UU., 20th Century Fox, Marvel Enterprises, Donners' Company, Bad Hat Harry Productions

Avellan, E., Bespalov, M., Bespalov, S., Boyko, O., Burlingham, A., ENGlebardt, S., EWingr, M. P., FieldS, A., GOdFreY, W. M., HADIDA, S., HADIDA, V., HAUSFATER, J., JAM, K., JOHNSONR, W. D., Kaufman, A., Klimov, A., l'Heureux, S., Madison, M., Manuel, M. C., Miller, F., Nelson, K., O'Neal, T., Proper, T., Rainone, F., Rodnyansky, A., Rodriguez, R., Smith, T., Stewart, A., Teterev, B., Thomas, S., Weinstein ,B., Weinstein, H. (productores), Miller, F., RODRIGUeZ, R. (directores). (2014). Sin City: Una dama por la que matar (Sin City: A Dame to Kill For). EE.UU., Aldamisa Entertainment, Demarest Films, Dimension Films, Miramax, Solipsist Film, The Fyzz Facility, The Weinstein Company, Troublemaker Studios.

Avellan, E., Scott, B., Weinstein, B., Weinstein, H. (productores), Miller, F., Rodriguez, R., TARAntino, Q. (directores). (2005). Sin City: Ciudad del pecado (Sin City). EE.UU., Dimension Films, Troublemaker Studios.

Avnet, J., Breton, B., De laurentils, A., De laurentils, R., Frost, S., Gordon, R., Haber, B., HaRgett, H., LAW, J., Oglesby, M. (productores), KeRRY CONRAN (director). (2004). Sky Captain y el mundo del mañana (Sky Captain and the World of Tomorrow), EE.UU., vino Unido, Italia, Paramount Pictures, Brooklyn Films II, Riff Raff Film Productions, Blue Flower Productions, Filmauro, Natural Nylon Entertainment.

Bartelme, J., Crowley, P., DAVISON, J., TiPPETt, P. (productores), KeRShner, I. (director). (1990). RoboCop 2 (RoboCop 2). EE.UU., Orion Pictures.

Bartelme, J., Crowley, P., La Marca, A. G. (productores), DekKer, F. (director). (1993). RoboCop 3 (RoboCop 3). EE.UU., Orion Pictures.

BLANKE, H., WALLIS, HAL, B. (productores), HOUSTON, J. (director) (1941). El halcón Maltés (The Maltese Falcon) EE UU., Warner Bros.

Burnett, A., Fukuda, N., Jeun, M., Melniker, B., Radomski, E., Ruegger, T., SeO, K., Timm, B., Uslan, M. E., Youn, J. (productores), RAdOMSKI, E., TIMM, B., AltierI, K., KIRKLAND, B., PAUR, F., RIBA, D. (directores). (1993). Batman: La mascara del fantasma (Batman: Mask of the Phantasm). EE.UU., Warner Bros. Animation.

Burnett, A., HiRuma, T., Macasero, M., Melniker, B., Register, S., Timm, B. Uslan, M. E. (productores), OLIVA, J. (director). (2012). Batman: El regreso del caballero oscuro. Primera parte (Batman: The Dark Knight Returns, Part 1). EE.UU., Warner Premiere, DC Entertainment, Warner Bros. Animation.

Burnett, A., HiRuma, T., Macasero, M., Melniker, B., Register, S., Timm, B. Uslan, M. E. (productores), OLIVA, J. (director). (2013). Batman: El regreso del caballero oscuro. Segunda parte (Batman: The Dark Knight Returns, Part 2). EE.UU., Warner Premiere, DC Entertainment, Warner Bros. Animation. 
Burnett, A., Carlin, M., Macasero, M., Melniker, B., Montgomery, L., Register, S., Timm, B., USLAN, M. E. (productores), LIU, S., MONTGOMERY, L. (directores). (2011). Batman: Año uno (Batman: Year One). EE.UU., Warner Premiere, DC Entertainment, Warner Bros. Animation.

Canton, S.B., Coller, W., Ewing, M. P., Flores, C. F., Garcia, A., Goldmann, Jashni, J., Jones, S., Miller, F., NunNari, G., Sharon, R., SNyDER, D., ThOMAS, S., Tull (productores), MURRO, N. (director). (2014). 300: El origen de un imperio (300: Rise of an Empire). EE.UU., Warner Bros., Legendary Entertainment, Cruel \& Unusual Films, Atmosphere Entertainment MM, Hollywood Gang Productions, Nimar Studios.

Canton, S.B., Coller, W., Fay, W., Flores, C. F., Goldmann, B., Mednick, S., Miller, F., Nunnari, G., Perrotta, J., Peter-Contesse, N., Silver, J., Snyder, D., Thomas, S., Tull, T., WAISBREN, B. (productores), SNYDER, Z. (director). 2006. 300 (300). EE.UU., Canadá, Bulgaria, Warner Bros., Legendary Entertainment, Virtual Studios, Hollywood Gang Productions, Atmosphere Entertainment MM, Mel's Cite du Cinema, Nimar Studios, Pixar Animation Studios.

Christie, T., Coller, W., Goyer, D. S., Johns, G., Johnson, W., Kanemoto, C., Melniker, B., Mnuchin, S., Moriarty, S., Nolan, C., Roven, C., Rowe, J., Snyder, D., Thomas, E., USLAN, M. E., WeRTheim, A., Wilson, G. (productores), SNYDER, Z. (director) (2016). Batman v Superman: El amanecer de la Justicia (Batman v Superman: Dawn of Justice). EE.UU., Warner Bros., Atlas Entertainment, Cruel \& Unusual Films, DC Comics, DC Entertainment, RatPac-Dune Entertainment, Zak Productions.

Cummings, D. (productor y director). (1987). The Making of 'RoboCop', EE.UU., Cummings Entertainment Corp.

Dauterive, M. E., MACGRegor-SCott, P., Melniker, B., AsLAN, M. E. (productores), Schumacher, J. (director). (1997). Batman y Robin (Batman \& Robin). EE.UU., Reino Unido, Warner Bros., PolyGram Filmed Entertainment.

Davison, J., LIM S., NeumeIER, E.,SCHMidT, A., TIPPETT, P. (productores). VerHOEVEN, P. (director). (1987). RoboCop (RoboCop), EE.UU., Orion Pictures.

FELDMAN P., SICKNER, R. N. (productores), PECKINPAH, S. (director). (1969). Grupo salvaje (The Wild Bunch), EE.UU., Warner Bros./Seven Arts.

franco, L., Melniker, B., Orleans, L., Roven, C., Thomas, E., TKach, C. A., Uslan, M. E. (productores), NOLAN, C. (director). (2005). Batman Begins (Batman Begins). EE.UU., Warner Bros., Syncopy, DC Comics, Legendary Entertainment, Patalex III Productions Limited.

FRIEDKIN, W., SMITH, B. S. (productores), FRIEDKIN, W. (director). (1977). Carga maldita (Sorcerer). EE.UU., Film Properties International N.V., Paramount Pictures, Universal Pictures.

Lippincott, C. M., Marks, B., Munafo, T., Nicoletti, S., Pressman, E. R., Vajna, A. G. (productores), CANNON, D. (director). (1995). Juez Dredd (Judge Dredd). EE.UU., Hollywood Pictures, Cinergi Pictures Entertainment, Edward R. Pressman Productions.

Martinelly, G, Thomas, J. (productores), CronenberG, D. (director). (1991). El almuerzo desnudo (The Naked Lunch), Canada, Reino Unido, Japón, Recorded Picture Company (RPC), Téléfilm Canada, Ontario Film Development Corporation, Film Trustees Ltd., Pierson, Heldring \& Pierson N.V., Nippon Film Development and Finance, Naked Lunch Productions.

MAtÉ, R., St. GeORge, G. (productores), MATÉ, R. (director). (1962). El león de Esparta (The 300 Spartans). EE.UU., 20th Century Fox. 
WALSH, B. (productor), STEVEnson, R. (director). (1964). Mary Poppins (Mary Poppins), EE.UU., Walt Disney Productions.

B) Televisión

AleXAnder, H., Burnett, A., Dini, P., MaCCurdy, J., TImm, B. (productores). (1997). Las nuevas aventuras de Batman (The New Batman Adventures). EE.UU., Warner Bros. Animation, DC Comics, Warner Bros. Television.

Bell, J., Brown, G. A., Lee, S., Loeb, J., TANChARoen, M., Whedon, J. Whedon, J. (productores) (2013). Agentes de S.H.I.E.L.D. (Agents of S.H.I.E.L.D.). EE.UU., ABC Studios, Marvel Television, Mutant Enemy.

C) Guiones no rodados

MILLER, F. (escritor). Batman: Year One (2002). http://leonscripts.users5.50megs.com/scripts/ BATMANYEARONEscript.htm 